\title{
Durable clinical benefit from PARP inhibition in a platinum-sensitive, BRCA2-mutated pancreatic cancer patient after earlier progression on placebo treatment on the POLO trial: a case report
}

\author{
Douglas Rubinson ${ }^{1}$, Brian M. Wolpin ${ }^{1}$, Ilana S. Warsofsky ${ }^{2}$, David P. Ryan ${ }^{3}$, Kimberly Perez ${ }^{1}$, \\ Osama Rahma ${ }^{1}$, Harshabad Singh ${ }^{1}$, Matthew B. Yurgelun ${ }^{1}$, Geoffrey I. Shapiro ${ }^{1}$, Andrew J. Aguirre ${ }^{1}$, \\ Alan D. D'Andrea ${ }^{1,4}$, James M. Cleary ${ }^{1}$ \\ ${ }^{1}$ Department of Medical Oncology, Dana-Farber Brigham and Women's Cancer Center, Dana-Farber Cancer Institute and Harvard Medical School, \\ Boston, MA, USA; ${ }^{2}$ Department of Radiology, Dana-Farber Brigham and Women's Cancer Center, Dana-Farber Cancer Institute and Harvard \\ Medical School, Boston, MA, USA; ${ }^{3}$ Department of Hematology/Oncology, Massachusetts General Hospital Cancer Center, Harvard Medical \\ School, Boston, MA, USA; ${ }^{4}$ Center for DNA Damage and Repair, Dana-Farber Cancer Institute, Boston, MA, USA \\ Correspondence to: James M. Cleary, MD, PhD. Dana-Farber Cancer Institute, 450 Brookline Ave, Boston, MA 02215, USA. \\ Email: james_cleary@dfci.harvard.edu.
}

\begin{abstract}
Metastatic pancreatic adenocarcinoma is a deadly malignancy with limited treatment options. Based on the results of the phase 3 POLO trial, the PARP inhibitor olaparib was approved by the Food and Drug Administration as a maintenance therapy in germline BRCA1- and BRCA2-mutated metastatic pancreatic cancer patients whose cancers had not progressed on first-line platinum-based chemotherapy. While this approval was a step forward, there have been criticisms of the POLO study leaving doubts in the field about the effectiveness of PARP inhibition in pancreatic cancer. Here, we describe a patient with a germline $B R C A 2$-mutated, metastatic pancreatic cancer who was randomized to the placeboarm of the POLO trial. After progressing on the placebo-arm of the POLO study, her cancer again responded to platinum-based chemotherapy and has since been successfully treated for 4 years with offprotocol maintenance olaparib. The presence of placebo treatment in this case serves as an internal control demonstrating the efficacy of PARP inhibition in this patient. This case highlights the potential of PARP inhibitor maintenance therapy in appropriately selected metastatic pancreatic cancer patients.
\end{abstract}

Keywords: Pancreatic cancer; PARP inhibitor; DNA repair; targeted therapy; case report

Submitted Apr 10, 2021. Accepted for publication Oct 26, 2021.

doi: 10.21037/jgo-21-197

View this article at: https://dx.doi.org/10.21037/jgo-21-197

\section{Introduction}

Metastatic pancreatic ductal adenocarcinoma (PDAC) is a nearly uniformly fatal disease with limited treatment options (1). Combinations of cytotoxic chemotherapies, such as 5-fluorouracil (5-FU), leucovorin, irinotecan, and oxaliplatin (FOLFIRINOX) and gemcitabine/nabpaclitaxel, have improved outcomes, but the median overall survival time for metastatic PDAC patients is still less than one year (2). Efforts to develop targeted and immunotherapeutic treatments for PDAC have been stymied by the high prevalence of KRAS mutations and its immunologically "cold" nature (2).

Large-scale genomic studies on germline and somatic DNA, have demonstrated that a subpopulation of PDAC patients have germline or somatic alterations in genes in the homologous recombination (HR) DNA repair pathway (3-5). Approximately $12 \%$ of pancreatic cancer patients possess a mutation within one of the "core HR genes" which cooperate in the Fanconi Anemia-BRCA pathway—BRCA1, 
BRCA2 and PALB2 $(3,5,6)$. Pancreatic cancers harboring HR gene mutations are more sensitive to platinum-based chemotherapy $(5,6)$. Importantly, patients with PDAC tumors harboring HR gene mutations, who are treated with platinum-based chemotherapy, have improved survival compared to similarly treated PDAC patients with tumors that do not harbor HR gene mutations $(5,6)$.

Pharmacological targeting of deleterious HR gene mutations started in 2005, when two laboratories observed synthetic lethality in cancer cells with homozygous BRCA mutations that were treated with poly (adenosine diphosphate-ribose) polymerase (PARP) inhibitors $(7,8)$. Subsequent clinical studies demonstrated the therapeutic applicability of this result, showing that orally available PARP inhibitors were well-tolerated and exhibited potent anti-tumor activity in HR-mutated cancers $(9,10)$. PARP inhibitors were first approved by the Food and Drug Administration (FDA) for the treatment of platinumsensitive $B R C A$-mutated ovarian cancer. Clinical trials demonstrated that three different PARP inhibitorsolaparib, niraparib, and rucaparib-improved progressionfree survival by $12-36$ months compared to placebo control in the maintenance setting (11-14). PARP inhibitors have since been approved by the FDA for the treatment of HRmutated breast and prostate cancers $(15,16)$.

The increasing recognition that a subset of PDAC also harbors HR gene mutations inspired efforts to therapeutically exploit this molecular vulnerability (17). Early clinical trials made the important observation that, as in the case of ovarian cancer, PARP inhibitor responses in PDAC were seen almost exclusively in platinum-sensitive cancers (18-20). Building on this observation, the Phase III, randomized, placebo-controlled Pancreas Cancer Olaparib Ongoing (POLO) trial (NCT02184195) was designed to test olaparib as a maintenance therapy in germline $B R C A 1$ - and BRCA2-mutated metastatic PDAC patients whose cancers had not progressed on first-line platinumbased chemotherapy (21). Maintenance olaparib increased median progression-free survival by 3.6 months compared to placebo control, leading to FDA approval of maintenance olaparib for the treatment of germline $B R C A$-mutated metastatic pancreatic cancer in December 2019. Consistent with the results of the POLO trial, advanced pancreatic adenocarcinoma patients with germline and somatic BRCA1, $B R C A 2$, and $P A L B 2$ mutations treated with maintenance rucaparib had a median progression-free survival of 9.3 months in a single-arm Phase II clinical trial (22).

In this report, we describe a patient with germline
BRCA2-mutated, metastatic PDAC who has benefited from maintenance olaparib for over 4 years. Importantly, the patient's previous progression on the placebo arm of the POLO study serves as an internal control clearly demonstrating the benefit of maintenance PARP inhibition in this case.

We present the following case in accordance with the CARE reporting checklist (available at https://dx.doi. org/10.21037/jgo-21-197).

\section{Case presentation}

A 52-year-old woman with no significant past medical history presented with right lower quadrant abdominal pain. A computed tomography (CT) scan revealed a $3.3-\mathrm{cm}$ pancreatic body mass and a $2.5-\mathrm{cm}$ abdominal wall nodule, along with possible peritoneal carcinomatosis. A diagnostic laparoscopy visualized multiple peritoneal nodules. Biopsies of a peritoneal metastasis and the abdominal wall nodule demonstrated adenocarcinoma consistent with a pancreatic primary. Peritoneal washings were also positive for adenocarcinoma. Her serum CA 19-9 was 1,081 U/mL.

The patient was of Eastern European descent and was not of Ashkenazi Jewish ancestry. The patient's brother died of pancreatic adenocarcinoma at the age of 42. Germline genetic testing performed on the patient showed a $B R C A 2$ c.2808_2811delACAA alteration. Attempts to sequence the somatic DNA from her tumor were unsuccessful.

The patient was initially treated with first-line FOLFIRINOX (Figure 1). Her cancer responded well to FOLFIRINOX. Radiologically, there was an $89 \%$ reduction in her tumor volume, per Response Evaluation Criteria in Solid Tumors (RECIST) 1.1, with a $67 \%$ reduction in the size of her pancreatic mass and a complete response in the abdominal wall nodule (Figure 2). Her serum CA 19-9 nadired at $12 \mathrm{U} / \mathrm{mL}$. Because of neuropathy, oxaliplatin was stopped after cycle 10 . The patient continued on 5-FU/leucovorin/irinotecan briefly, but all chemotherapeutic treatments were stopped after cycle 12 because the patient wanted to recover from the cumulative toxicities of chemotherapy. Following a two-month break from chemotherapy, the patient elected to enroll in the double-blind, placebo-controlled POLO trial evaluating maintenance olaparib in germline $B R C A$-mutated patients. The patient remained in the POLO trial for 5 months. At the end of the 5 months, the patient experienced progressively worsening abdominal pain and her CA 19-9 levels climbed to $174 \mathrm{U} / \mathrm{mL}$ (Figure 1). Restaging CT scans 

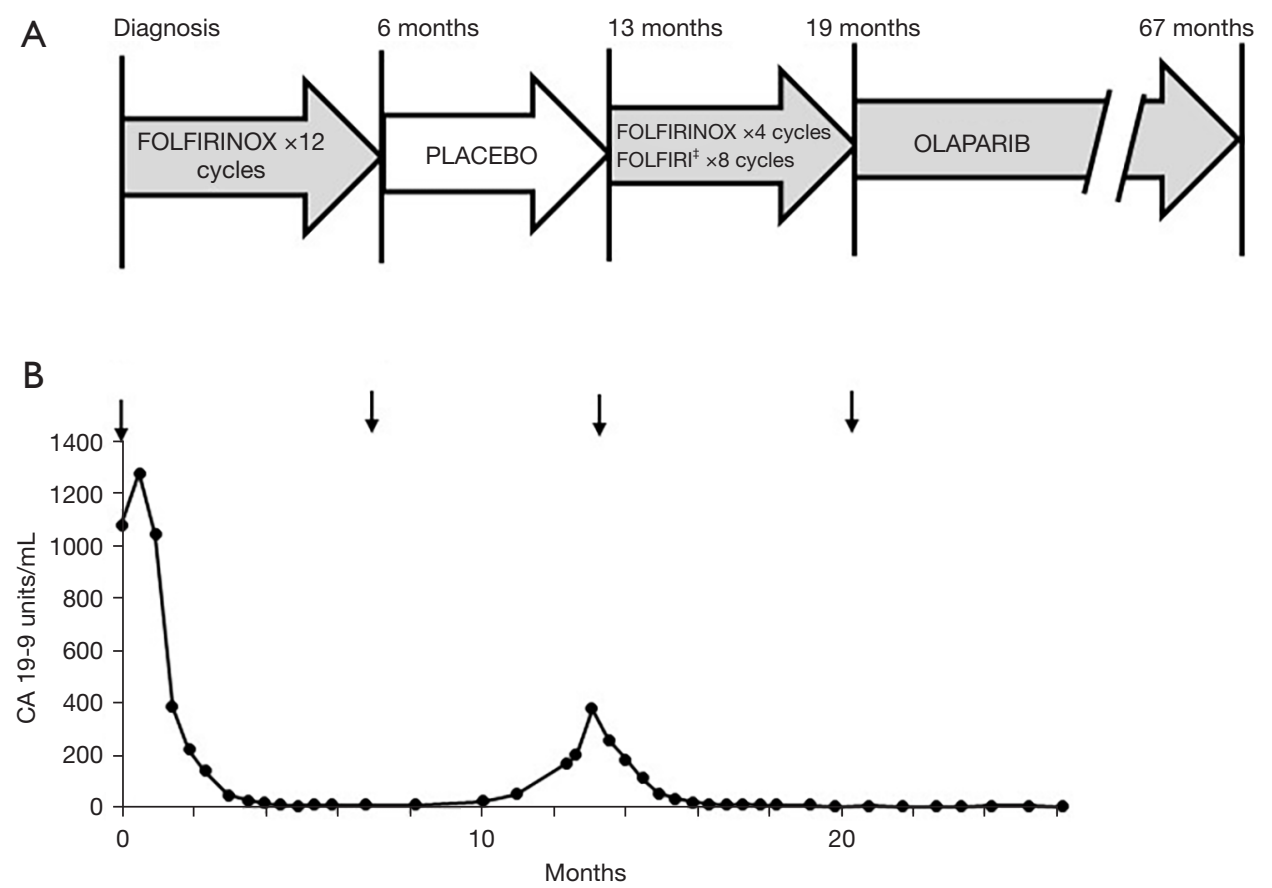

Figure 1 Treatment history and serological CA 19-9 response of a pancreatic cancer patient with a germline BRCA2 mutation. (A) Timeline depicting the treatment course and duration of therapy; (B) graphical depiction of serial CA 19-9 measurements over the course of treatment. Arrows correspond to time points when a new treatment was started in the clinical timeline presented in (A). FOLFIRI ${ }^{\ddagger}$ indicates 5-fluorouracil/liposomal irinotecan.

showed an increase in size of the pancreatic mass, and the patient was taken off the POLO trial (Figure 2).

The patient was subsequently re-treated with FOLFIRINOX. After cycle 4 of FOLFIRINOX, the oxaliplatin was stopped and the patient was continued on 5-FU/leucovorin/liposomal irinotecan. The patient's tumor again responded well to chemotherapy and CT scans demonstrated a radiologic complete response to therapy (Figure 2). Her serum CA 19-9 level fell to $14 \mathrm{U} / \mathrm{mL}$ (Figure 1). While the cancer responded well to chemotherapy, the patient increasingly struggled with the cumulative toxicities of therapy and requested a chemotherapy holiday after the $8^{\text {th }}$ cycle of chemotherapy.

A request was made to the sponsor of the POLO study to unblind the treatment the patient received, and it was revealed that she was in the placebo arm of the POLO study. The patient was then started on off-protocol olaparib. The patient tolerated the olaparib well with an excellent quality of life. As of this report, she has been on olaparib for 4 years. CT scans have continued to show a complete response and her CA 19-9 levels have been normal, with a range of $5-12 \mathrm{U} / \mathrm{mL}$, throughout the course of olaparib maintenance therapy (Figures 1,2).

All procedures performed in studies involving human participants were in accordance with the ethical standards of the institutional and/or national research committee(s) and with the Helsinki Declaration (as revised in 2013). Written informed consent was obtained from the patient.

\section{Discussion}

Metastatic PDAC has earned its reputation as a recalcitrant malignancy with limited treatment options and a dismal prognosis (1). In a step forward, the POLO trial demonstrated that olaparib maintenance therapy improved progression-free survival of patients with germline BRCA1and $B R C A 2$-mutated pancreatic adenocarcinoma (21). However, unlike the dramatic benefits seen in ovarian cancer patients, the median progression-free survival benefit for pancreatic cancer patients was 3.6 months (21). Furthermore, compared to placebo-treated patients, there was no improvement in overall survival in olaparib-treated PDAC patients $(21,23)$. One possible explanation for the modest progression-free survival benefit is that germline 


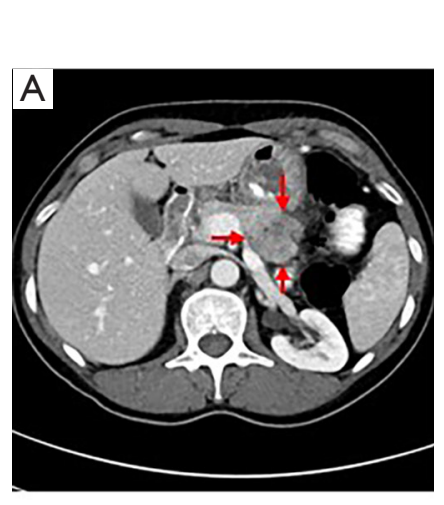

Baseline

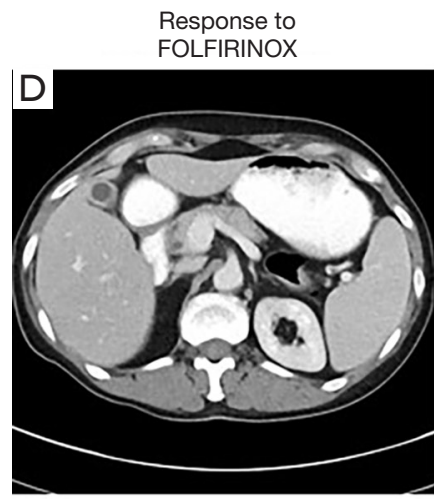

18 months

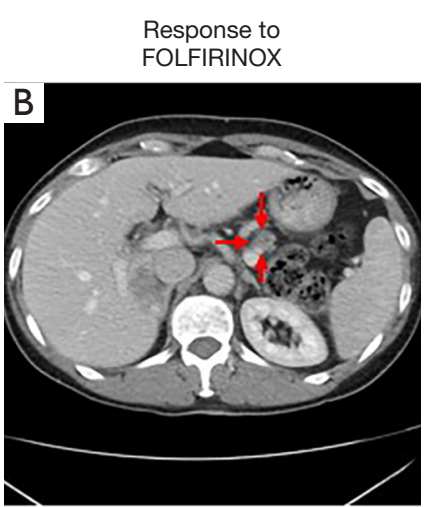

6 months

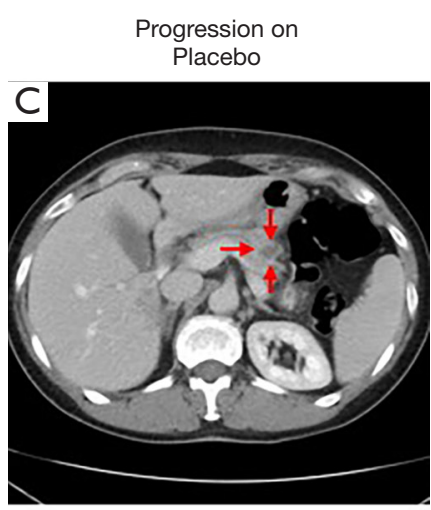

13 months

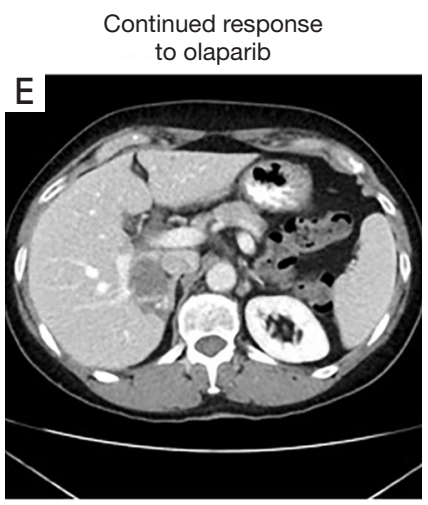

67 months

Figure 2 CT scans demonstrating the radiological response of the pancreatic tumor to therapy over the course of treatment. Arrows indicate the location of the pancreatic tumor. FOLFIRINOX, 5-fluorouracil (5-FU), leucovorin, irinotecan, and oxaliplatin; CT, computed tomography.

BRCA-mutated pancreatic cancer may continue to have sustained response following a response to platinum-based chemotherapy. Supporting this assertion is the observation that on the POLO trial the 24-month progression-free survival rate for placebo was $9.6 \%$. However, the patient described in this report had significant progression while receiving placebo with symptomatic, radiologic and biochemical progression after 5 months. Despite the aggressive proclivity of this patient's malignancy, she was able to benefit from a renewed response to platinum-based therapy and then enjoy a remarkably durable response to olaparib for over 4 years to date. In addition to her oncologic benefit, this patient has maintained a very good quality of life while receiving olaparib after previously abandoning multi-agent chemotherapy due to accumulated toxicity. Given her excellent tolerance on olaparib, the therapeutic plan is for her to continue on olaparib indefinitely as long as it continues to control her cancer.

In the POLO trial, pancreatic cancer patients who had a radiological partial response to maintenance olaparib had an impressive median duration of response of 24.9 months, compared to the 3.7-month median duration of response for the placebo group (21). However, while olaparib clearly had activity in the $20 \%$ of pancreatic cancer patients who achieved a partial response to PARP inhibition, the olaparibtreated group as a whole had only a modest 3.6-month median progression-free survival improvement over the placebo group (21). The disparity of clinical benefit documented in the POLO trial highlights the need for better clinical indicators to select pancreatic patients for maintenance PARP inhibitor therapy. While the POLO study resembles the PARP inhibitor maintenance strategy performed in ovarian cancer trials, the efficacy in these two disease populations is different (3.6 vs. 12-36 months, 
respectively) (11-14). An important difference between the pancreatic and ovarian cancer trials is how "platinumsensitive" is defined (11-14). In PARP inhibitor maintenance trials for ovarian cancer, "platinum-sensitive" referred to patients who had at least a partial response to platinumbased chemotherapy (11-14). The threshold for platinum sensitivity was lower in the POLO study; patients were only required to have no evidence of radiological disease progression following at least 16 weeks of platinum-based chemotherapy to be classified as having platinum-sensitive cancer (21). However, surprisingly, on the POLO trial, patients who achieved a radiological response to firstline platinum-based chemotherapy did not have superior progression-free survival compared to patients who had stable disease on first-line platinum-based chemotherapy. Future studies are needed to better define the radiological response parameters of platinum-based chemotherapy that most accurately predict a therapeutic response to PARP inhibitors in PDAC patients. We hypothesize that a deep radiological response to platinum-based chemotherapy, such as the $89 \%$ and $100 \%$ reduction in tumor volume in this patient's two courses of FOLFIRINOX, could be a more accurate biomarker for PARP inhibitor sensitivity in the BRCA-mutated pancreatic cancer population.

While the POLO trial only analyzed pancreatic cancer patients with germline BRCA1 and BRCA/2 mutations, a recent trial of maintenance rucaparib has also demonstrated responses of pancreatic cancer patients with somatic $B R C A$ and PALB2 mutations (22). However, more research is needed to determine if tumors with germline HR gene mutations are more sensitive to PARP inhibition than tumors with somatic HR gene mutations. Germline HR gene mutations are often assumed to have greater PARP inhibitor sensitivity because there is a higher probability of biallelic inactivation, but this has not been empirically tested. Furthermore, prospective clinical data are needed to assess whether other predictive biomarkers of PARP inhibitor sensitivity, such as biallelic inactivation of $\mathrm{HR}$ genes, genomic HRD assays, genomic signature assays of signature 3, or functional assays of HR deficiency, are effective in selecting pancreatic cancer patients who will benefit from PARP inhibitor therapy $(6,24)$.

Germline BRCA2 mutations occur in up to $5 \%$ of pancreatic cancer patients and are the most common type of HR gene mutations $(5,6,25)$. The $B R C A 2$ c.2808_2811delACAA mutation found in this patient is one of the most common BRCA2 mutations in nonAshkenazi Jews with breast cancer (26). The frameshift mutation occurs in the RAD51-binding domain of BRCA2 on exon 11 and causes a truncated BRCA2 protein. An important function of BRCA2 is binding to DNA doublestrand breaks and acting as a scaffold for the formation of RAD51 filaments, which mediate DNA repair through homologous recombination (27). Interestingly, LabidiGaly et al. reported that among ovarian cancer patients with germline $B R C A 2$ mutations, patients with germline exon $11 B R C A 2$ mutations had improved survival compared to ovarian cancer patients who were not carriers of BRCA2 germline mutations (28). In a case series examining PARP inhibitor sensitivity in pancreatic cancer patients, Borazanci et al. found that 4 pancreatic cancer patients, who were carriers of germline $B R C A 1$ or $B R C A 2$ mutations involving the RAD51-binding domain, were particularly sensitive to PARP inhibitors, and had a median overall survival time of 24 months (29). Further studies are needed to establish if BRCA2 mutations involving the RAD51-binding domain are predictive of increased sensitivity to PARP inhibition.

In conclusion, this case highlights that platinumsensitive, germline $B R C A$-mutated patients with advanced PDAC can be exquisitely sensitive to PARP inhibition. Despite progressing on placebo in the POLO trial, the patient had a substantial radiological response to two separate courses of FOLFIRINOX and benefited from olaparib maintenance therapy for 4 years. Future studies are needed to better identify predictive biomarkers that can effectively distinguish pancreatic cancers that are sensitive to PARP inhibition.

\section{Acknowledgments}

Funding: This work was supported by the DFCI Hale Family Center for Pancreatic Cancer (BMW, AA, JMC), Stand Up To Cancer (BMW, GIS, ADD, JMC), the Lustgarten Foundation (BMW, GIS, AA, ADD, JMC) and NIH P50 CA127003, the Dana-Farber/Harvard Cancer Center Specialized Program of Research Excellence (SPORE) in Gastrointestinal Cancer (BMW, GIS, AA, ADD, JMC). The POLO trial was funded by Astrazeneca. BMW is also supported by NIH U01 CA210171, Noble Effort Fund, Wexler Family Fund, and Promises for Purple. HS is a William Raveis Charitable Fund Physician-Scientist of the Damon Runyon Cancer Research Foundation (PST15-18). AJA is also supported by the Doris Duke Charitable Foundation, Pancreatic Cancer Action Network, NIH-NCI K08 CA218420-02, U01 CA224146, and U01 CA250549. JMC is also supported by the Haya Linde Memorial Fund, 
Team Evan Schumacher, and the Grateful Foundation.

\section{Footnote}

Reporting Checklist: The authors have completed the CARE reporting checklist. Available at https://dx.doi. org/10.21037/jgo-21-197

Peer Review File: Available at https://dx.doi.org/10.21037/ jgo-21-197

Conflicts of Interest: All authors have completed the ICMJE uniform disclosure form (available at https://dx.doi. org/10.21037/jgo-21-197). DR reports personal fees from Boston Scientific, outside the submitted work. BMW receives grant support from Celgene and Eli Lilly, and has consulted for BioLineRx, Celgene and GRAIL. DPR reports grants from Astrazeneca, during the conduct of the study; grants from SU2C, personal fees from EXAS/Thrive, personal fees from MPM, personal fees from Boeringer Ingelheim, personal fees from UpToDate, outside the submitted work. OR reports personal fees from Astrazeneca, during the conduct of the study; grants and personal fees from Merck, personal fees from Celgene, personal fees from Five Prime, personal fees from GSK, personal fees from Bayer, personal fees from Roche/Genentech, personal fees from Puretech, personal fees from Imvac, personal fees from Sobi, outside the submitted work; In addition, Dr. Rahma has a patent Methods of using pembrolizumab and trebananib pending. HS is a William Raveis Charitable Fund Physician-Scientist of the Damon Runyon Cancer Research Foundation (PST-15-18). MBY reports grants and personal fees from Janssen Pharmaceuticals, personal fees from UpToDate, outside the submitted work. GIS reports grants from Eli Lilly, grants and personal fees from Merck KGaA/EMD Serono, personal fees from Merck \& Co., grants and personal fees from Sierra Oncology, personal fees from Pfizer, personal fees from G1 Therapeutics, personal fees from Bicycle Therapeutics, personal fees from Roche, personal fees from Fusion Pharmaceuticals, personal fees from Cybrexa Therapeutics, personal fees from Astex, personal fees from Almac, personal fees from Ipsen, personal fees from Bayer, personal fees from Angiex, personal fees from Daiichi Sankyo, personal fees from Seattle Genetics, personal fees from Boehringer Ingelheim, personal fees from ImmunoMet, personal fees from Asana, personal fees from Artios, personal fees from Atrin, personal fees from Concarlo Holdings, personal fees from Syros, personal fees from Zentalis, personal fees from CytomX Therapeutics, personal fees from Blueprint Medicines, outside the submitted work; In addition, Dr. Shapiro has a patent Dosage regimen for sapacitabine and seliciclib issued to Cyclacel Pharmaceuticals and Geoffrey Shapiro, and a patent Compositions and Methods for Predicting Response and Resistance to CDK4/6 inhibition pending to Liam Cornell and Geoffrey Shapiro. AJA reports grants from National Cancer Institute, grants from Lustgarten Foundation, grants from Pancreatic Cancer Action Network, grants from Doris Duke Charitable Foundation, grants from Dana-Farber Cancer Institute Hale Center for Pancreatic Cancer Research, during the conduct of the study; grants from Mirati Therapeutics, personal fees from Merck, personal fees from Arrakis Therapeutics, personal fees from Oncorus, grants from Deerfield, Inc., grants from Novo Ventures, grants and personal fees from Syros Pharmaceuticals, outside the submitted work. ADD reports personal fees from Bayer AG, personal fees and other from Cedilla Therapeutics, personal fees and other from Cyteir Therapeutics, personal fees from Epizyme, Inc., personal fees from GalaxoSmithKline, personal fees and other from Ideaya, Inc., personal fees from Impact Therapeutics, personal fees from LAV Global Management, personal fees from L.E.K. Consulting, grants and personal fees from Lilly Oncology, grants and personal fees from Merck KGAa/ EMD Serono, personal fees from Novartis Bioventures, personal fees from Novo Ventures, personal fees from Pfizer, personal fees from Tango Therapeutics, outside the submitted work. JMC reports non-financial support from AZD, grants from Abbvie, grants from Merus, grants from Roche, grants from BMS, grants from Merck, non-financial support from Esperas Pharma, grants from Tesaro, personal fees from BMS, from Syros, outside the submitted work. The other authors have no conflicts of interest to declare.

Ethical Statement: The authors are accountable for all aspects of the work in ensuring that questions related to the accuracy or integrity of any part of the work are appropriately investigated and resolved. All procedures performed in studies involving human participants were in accordance with the ethical standards of the institutional and/or national research committee(s) and with the Helsinki Declaration (as revised in 2013). Written informed consent was obtained from the patient.

Open Access Statement: This is an Open Access article distributed in accordance with the Creative Commons 
Attribution-NonCommercial-NoDerivs 4.0 International License (CC BY-NC-ND 4.0), which permits the noncommercial replication and distribution of the article with the strict proviso that no changes or edits are made and the original work is properly cited (including links to both the formal publication through the relevant DOI and the license). See: https://creativecommons.org/licenses/by-nc-nd/4.0/.

\section{References}

1. Mizrahi JD, Surana R, Valle JW, et al. Pancreatic cancer. Lancet 2020;395:2008-20.

2. Singh RR, O'Reilly EM. New Treatment Strategies for Metastatic Pancreatic Ductal Adenocarcinoma. Drugs 2020;80:647-69.

3. Aguirre AJ, Nowak JA, Camarda ND, et al. Real-time Genomic Characterization of Advanced Pancreatic Cancer to Enable Precision Medicine. Cancer Discov 2018;8:1096-111.

4. Jones S, Zhang X, Parsons DW, et al. Core signaling pathways in human pancreatic cancers revealed by global genomic analyses. Science 2008;321:1801-6.

5. Yurgelun MB, Chittenden AB, Morales-Oyarvide V, et al. Germline cancer susceptibility gene variants, somatic second hits, and survival outcomes in patients with resected pancreatic cancer. Genet Med 2019;21:213-23.

6. Park W, Chen J, Chou JF, et al. Genomic Methods Identify Homologous Recombination Deficiency in Pancreas Adenocarcinoma and Optimize Treatment Selection. Clin Cancer Res 2020;26:3239-47.

7. Bryant HE, Schultz N, Thomas HD, et al. Specific killing of BRCA2-deficient tumours with inhibitors of poly(ADPribose) polymerase. Nature 2005;434:913-7.

8. Farmer H, McCabe N, Lord CJ, et al. Targeting the DNA repair defect in BRCA mutant cells as a therapeutic strategy. Nature 2005;434:917-21.

9. Fong PC, Boss DS, Yap TA, et al. Inhibition of poly(ADPribose) polymerase in tumors from BRCA mutation carriers. N Engl J Med 2009;361:123-34.

10. Kaufman B, Shapira-Frommer R, Schmutzler RK, et al. Olaparib monotherapy in patients with advanced cancer and a germline BRCA1/2 mutation. J Clin Oncol 2015;33:244-50.

11. Pujade-Lauraine E, Ledermann JA, Selle F, et al. Olaparib tablets as maintenance therapy in patients with platinumsensitive, relapsed ovarian cancer and a BRCA1/2 mutation (SOLO2/ENGOT-Ov21): a double-blind, randomised, placebo-controlled, phase 3 trial. Lancet
Oncol 2017;18:1274-84.

12. Coleman RL, Oza AM, Lorusso D, et al. Rucaparib maintenance treatment for recurrent ovarian carcinoma after response to platinum therapy (ARIEL3): a randomised, double-blind, placebo-controlled, phase 3 trial. Lancet 2017;390:1949-61.

13. Mirza MR, Monk BJ, Herrstedt J, et al. Niraparib Maintenance Therapy in Platinum-Sensitive, Recurrent Ovarian Cancer. N Engl J Med 2016;375:2154-64.

14. Moore K, Colombo N, Scambia G, et al. Maintenance Olaparib in Patients with Newly Diagnosed Advanced Ovarian Cancer. N Engl J Med 2018;379:2495-505.

15. de Bono J, Mateo J, Fizazi K, et al. Olaparib for Metastatic Castration-Resistant Prostate Cancer. N Engl J Med 2020;382:2091-102.

16. Litton JK, Rugo HS, Ettl J, et al. Talazoparib in Patients with Advanced Breast Cancer and a Germline BRCA Mutation. N Engl J Med 2018;379:753-63.

17. Cleary JM, Wolpin BM, Dougan SK, et al. Opportunities for Utilization of DNA Repair Inhibitors in Homologous Recombination Repair-Deficient and Proficient Pancreatic Adenocarcinoma. Clin Cancer Res 2021;27:6622-37.

18. Shroff RT, Hendifar A, McWilliams RR, et al. Rucaparib Monotherapy in Patients With Pancreatic Cancer and a Known Deleterious BRCA Mutation. JCO Precis Oncol 2018;2018:PO.17.00316.

19. Lowery MA, Kelsen DP, Capanu M, et al. Phase II trial of veliparib in patients with previously treated BRCAmutated pancreas ductal adenocarcinoma. Eur J Cancer 2018;89:19-26.

20. Fong PC, Yap TA, Boss DS, et al. Poly(ADP)-ribose polymerase inhibition: frequent durable responses in BRCA carrier ovarian cancer correlating with platinumfree interval. J Clin Oncol 2010;28:2512-9.

21. Golan T, Hammel P, Reni M, et al. Maintenance Olaparib for Germline BRCA-Mutated Metastatic Pancreatic Cancer. N Engl J Med 2019;381:317-27.

22. Binder KAR, Mick R, O'Hara M, et al. Abstract CT234: A Phase II, single arm study of maintenance rucaparib in patients with platinum-sensitive advanced pancreatic cancer and a pathogenic germline or somatic mutation in BRCA1, BRCA2 or PALB2. Conference: Proceedings: AACR Annual Meeting 2019. DOI: 10.1158/1538-7445. AM2019-CT234.

23. Golan T, Hammel P, Reni M, et al. Overall survival from the phase 3 POLO trial: Maintenance olaparib for germline BRCA-mutated metastatic pancreatic cancer. J 
Clin Oncol 2021;39:abstr 378.

24. Cleary JM, Aguirre AJ, Shapiro GI, et al. BiomarkerGuided Development of DNA Repair Inhibitors. Mol Cell 2020;78:1070-85.

25. Wong W, Raufi AG, Safyan RA, et al. BRCA Mutations in Pancreas Cancer: Spectrum, Current Management, Challenges and Future Prospects. Cancer Manag Res 2020;12:2731-42.

26. Infante $M$, Durán $M$, Acedo A, et al. The highly prevalent BRCA2 mutation c.2808_2811del (3036delACAA) is located in a mutational hotspot and has multiple origins.

Cite this article as: Rubinson D, Wolpin BM, Warsofsky IS, Ryan DP, Perez K, Rahma O, Singh H, Yurgelun MB, Shapiro GI, Aguirre AJ, D'Andrea AD, Cleary JM. Durable clinical benefit from PARP inhibition in a platinum-sensitive, BRCA2-mutated pancreatic cancer patient after earlier progression on placebo treatment on the POLO trial: a case report. J Gastrointest Oncol 2021;12(6):3133-3140. doi: 10.21037/ jgo-21-197
Carcinogenesis 2013;34:2505-11.

27. Holloman WK. Unraveling the mechanism of BRCA2 in homologous recombination. Nat Struct Mol Biol 2011;18:748-54.

28. Labidi-Galy SI, Olivier T, Rodrigues M, et al. Location of Mutation in BRCA2 Gene and Survival in Patients with Ovarian Cancer. Clin Cancer Res 2018;24:326-33.

29. Borazanci E, Korn R, Liang WS, et al. An Analysis of Patients with DNA Repair Pathway Mutations Treated with a PARP Inhibitor. Oncologist 2020;25:e60-7. 